MATHEMATICS OF COMPUTATION

Volume 66, Number 220, October 1997, Pages 1645-1662

S 0025-5718(97)00854-5

\title{
ON THE SINGULAR VALUES OF WEBER MODULAR FUNCTIONS
}

\author{
NORIKO YUI AND DON ZAGIER
}

\begin{abstract}
The minimal polynomials of the singular values of the classical Weber modular functions give far simpler defining polynomials for the class fields of imaginary quadratic fields than the minimal polynomials of singular moduli of level 1. We describe computations of these polynomials and give conjectural formulas describing the prime decomposition of their resultants and discriminants, extending the formulas of Gross-Zagier for the level 1 case.
\end{abstract}

\section{INTRODUCTION}

Singular moduli, the values of the elliptic modular function of level one $j(\tau)$ at imaginary quadratic arguments $\tau$ in the upper half complex plane $\mathfrak{H}$, were studied extensively by many mathematicians in the early part of this century [11], [10], [2]. Their most important application is to explicit class field theory: if $K=$ $\mathbb{Q}(\sqrt{d})$ is an imaginary quadratic field of discriminant $d$, then the numbers $j(\tau)$, where $\tau$ ranges over the $S L_{2}(\mathbb{Z})$-inequivalent points in $\mathfrak{H}$ satisfying a quadratic equation of discriminant $d$, are the roots of a computable polynomial $H_{d}(X) \in \mathbb{Z}[X]$, the class polynomial. Since each root generates the Hilbert class field of $K$ when adjoined to $K$, this gives an explicit way to construct class fields. There are other applications, e.g. to the problems of primality testing/proving [1] and to the study of representability of primes by quadratic forms $x^{2}+n y^{2}[5]$.

A drawback of the polynomials $H_{d}(X)$, however, is that they have coefficients of astronomical size even for quite modest discriminants $d$, e.g., $H_{-55}(X)$ is

$$
\begin{aligned}
X^{4} & +3^{3} 5^{3} 29 \cdot 134219 X^{3}-3^{7} 5^{3} 23 \cdot 101 \cdot 32987 X^{2} \\
& +3^{9} 5^{7} 11^{2} 83 \cdot 101 \cdot 110641 X-3^{12} 5^{6} 11^{3} 29^{3} 41^{3} .
\end{aligned}
$$

In this paper we shall look at the class equations obtained by using the Weber function $\mathfrak{f}(\tau)$ of level 48 and index 72 instead of the modular invariant $j(\tau)$ of level 1. We will concentrate on the case of discriminants $d$ congruent to 1 modulo 8 and not divisible by 3 , since the results are optimal here: the values of $\mathfrak{f}$ at suitable points $\tau \in \mathfrak{H}$ of discriminant $d$ generate the same fields as before but are the roots of a polynomial $W_{d}(X)$ having far smaller coefficients than $H_{d}(X)$. For instance, $W_{-55}(X)$ is simply $X^{4}+X^{3}-2 X-1$.

Our first aim will be to give an efficient procedure for calculating the roots of the Weber class polynomials $W_{d}(X)$ by describing a rule for picking out of each $S L_{2}(\mathbb{Z})$-equivalence class of points $\tau \in \mathfrak{H}$ of discriminant $d$ one $\Gamma$-equivalence class,

Received by the editor June 8, 1994 and, in revised form, June 19, 1996.

1991 Mathematics Subject Classification. Primary 11G15; Secondary 11R37, 11F03, 11G16.

(C)1997 American Mathematical Society 
where $\Gamma \subset S L_{2}(\mathbb{Z})$ is the subgroup of index 72 under which $\mathfrak{f}(\tau)$ is invariant, in such a way that the corresponding values of $\mathfrak{f}(\tau)$ are the roots of $W_{d}(X)$. Our second, and more important, objective is to give analogues for the Weber function of the results of Gross-Zagier [7] on the norms of the differences of singular moduli $j(\tau)$, i.e., on the discriminants and resultants of the class polynomials $H_{d}(X)$. The result of [7], following earlier work of Deuring [6], was that the norms in question are highly factorizable and that there is a closed formula for their decomposition into prime powers. The corresponding numbers for the Weber polynomials are far smaller - for instance, the discriminant of the polynomial $H_{-55}$ given above is

$$
-3^{78} 5^{20} 11^{5} 19^{6} 23^{6} 29^{2} 37^{2} 41^{2} 47^{4} 53^{2} \approx-4.7 \times 10^{91},
$$

while that of $W_{-55}(X)$ is only $-5^{2} 11=-275$ - but the rule for finding their prime factors and the exponents to which they occur turns out to be considerably more complicated. More precisely, the formula given in [7] for the prime factorization of norms of differences $j\left(\tau_{1}\right)-j\left(\tau_{2}\right)$ involved a certain arithmetic function $\mathfrak{F}(m)$ which takes on prime power values. It turns out that $\mathfrak{F}(m)$ decomposes naturally into the product of 8 more complicated functions $\mathfrak{F}_{r}(m)$ (where $r$ runs over the divisors of 24) which can be used in a similar way to describe the norms of differences of singular values of $\mathfrak{f}(\tau)^{r}$. This result is conjectural only, based on extensive numerical computations; it could presumably be proved by methods analogous to those of [7], but we have not tried to do this. The formula describing the function $\mathfrak{F}_{r}(m)$, given in $\S 3$, is complicated and involves many case distinctions, the necessary data being given in two tables with a total of 48 entries. In $\S 4$ we transform the conjecture into a different form which still involves some arbitrary data but does not need the large number of case distinctions. In both $\S 3$ and $\S 4$ we study the differences of Weber singular moduli corresponding to different quadratic fields (giving the resultants of different Weber polynomials). The differences of singular moduli for the same quadratic field (giving the discriminants of Weber polynomials) are considered in $\S 5$. The final section contains a brief discussion of the cases when $d$ is not congruent to 1 modulo 8 or is divisible by 3 , so that the Weber singular moduli belong to a small extension of the Hilbert class field.

\section{The Weber functions and Weber class equations}

For $\tau \in \mathfrak{H}$ (upper half-plane) we set $q=e^{2 \pi i \tau}$ and more generally $q^{a}=e^{2 \pi i a \tau}$ $(a \in \mathbb{Q})$. The classical Weber functions are defined by

$$
\begin{gathered}
\mathfrak{f}(\tau)=q^{-\frac{1}{48}} \prod_{n=1}^{\infty}\left(1+q^{n-\frac{1}{2}}\right), \quad \mathfrak{f}_{1}(\tau)=q^{-\frac{1}{48}} \prod_{n=1}^{\infty}\left(1-q^{n-\frac{1}{2}}\right), \\
\mathfrak{f}_{2}(\tau)=\sqrt{2} q^{\frac{1}{24}} \prod_{n=1}^{\infty}\left(1+q^{n}\right) .
\end{gathered}
$$

The functions $\mathfrak{f}^{24},-\mathfrak{f}_{1}^{24}$ and $-\mathfrak{f}_{2}^{24}$ are the roots of $(X-16)^{3}-X j=0$, where $j=j(\tau)$ is the elliptic modular function. In particular, $S L_{2}(\mathbb{Z})$ preserves the set of functions $\left\{\mathfrak{f}, \mathfrak{f}_{1}, \mathfrak{f}_{2}\right\}$ up to permutation and multiplication by 48 th roots of unity. The action 
of the generators of $S L_{2}(\mathbb{Z})$ on these functions is given by

$$
\left(\begin{array}{c}
\mathfrak{f}(\tau+1) \\
\mathfrak{f}_{1}(\tau+1) \\
\mathfrak{f}_{2}(\tau+1)
\end{array}\right)=\left(\begin{array}{ccc}
0 & \zeta^{-1} & 0 \\
\zeta^{-1} & 0 & 0 \\
0 & 0 & \zeta^{2}
\end{array}\right)\left(\begin{array}{c}
\mathfrak{f}(\tau) \\
\mathfrak{f}_{1}(\tau) \\
\mathfrak{f}_{2}(\tau)
\end{array}\right), \quad\left(\begin{array}{c}
\mathfrak{f}(-1 / \tau) \\
\mathfrak{f}_{1}(-1 / \tau) \\
\mathfrak{f}_{2}(-1 / \tau)
\end{array}\right)=\left(\begin{array}{ccc}
1 & 0 & 0 \\
0 & 0 & 1 \\
0 & 1 & 0
\end{array}\right)\left(\begin{array}{c}
\mathfrak{f}(\tau) \\
\mathfrak{f}_{1}(\tau) \\
\mathfrak{f}_{2}(\tau)
\end{array}\right)
$$

where $\zeta=\zeta_{48}=e^{2 \pi i / 48}$. We will continue to use the classical notations $\mathfrak{f}, \mathfrak{f}_{1}, \mathfrak{f}_{2}$, but mention that a more uniform and less arbitrary notation for the Weber functions would be

$$
\mathfrak{f}_{\left(\begin{array}{c}
0 \\
1
\end{array}\right)}(\tau)=\mathfrak{f}_{1}(\tau), \quad \mathfrak{f}_{\left(\begin{array}{l}
1 \\
0
\end{array}\right)}(\tau)=\mathfrak{f}_{2}(\tau), \quad \mathfrak{f}_{\left(\begin{array}{l}
1 \\
1
\end{array}\right)}(\tau)=\zeta \mathfrak{f}(\tau),
$$

since: (i) the functions $\mathfrak{f}_{\xi}^{8}$ are conjugate over $\mathbb{C}\left(j^{1 / 3}\right)$ and the functions $\mathfrak{f}_{\xi}^{24}$ over $\mathbb{C}(j)$; (ii) $\mathfrak{f}_{\xi}(\tau)^{24}=\frac{2^{12}}{(c \tau+d)^{12}} \frac{\Delta(M \tau)}{\Delta(\tau)}$ where $\Delta(\tau)=q \prod\left(1-q^{n}\right)^{24}$ is the classical discriminant function and $M=\left(\begin{array}{ll}a & b \\ c & d\end{array}\right)$ any $2 \times 2$ integral matrix of determinant 2 with $M \xi \equiv 0(\bmod 2)$; and (iii) the transformation law becomes $\mathfrak{f}_{\xi}(\gamma \tau)=\lambda \mathfrak{f}_{\xi^{\prime}}(\tau)$ for any $\gamma \in S L_{2}(\mathbb{Z})$, where $\gamma \xi^{\prime} \equiv \xi(\bmod 2)$ and $\lambda$ is a 24 th root of unity.

We now define the Weber class invariants which we want to study. Consider $d$ such that

$$
d<0, \quad d \equiv 1 \quad(\bmod 8), \quad d \not \equiv 0 \quad(\bmod 3),
$$

i.e., $d$ is the discriminant of an order $\mathcal{O}$ in an imaginary quadratic field in which 2 splits and 3 is unramified. Let $\operatorname{Pic}(\mathcal{O})$ denote the group of ideal classes of $\mathcal{O}$. We will represent its elements by $S L_{2}(\mathbb{Z})$-equivalence classes $[Q]$ of primitive positive definite quadratic forms $Q=[a, b, c]$ of discriminant $b^{2}-4 a c=d$. To each $Q$ is associated the number $\tau_{Q}=(-b+\sqrt{d}) / 2 a$ which is the unique root in $\mathfrak{H}$ of $Q(\tau, 1)=0$. From the transformation formulas just given it is clear that the number $\mathfrak{f}_{\xi}\left(\tau_{Q}\right)^{24}$, where $\xi$ is the unique vector in $\mathbb{F}_{2}^{2}$ with $Q(\xi) \not \equiv 0(\bmod 2)$, depends only on the class $[Q]$. We now define a certain 24 th root of (the negative of) this number with the same invariance property.

Proposition. Let $d$ be a discriminant satisfying (1). For $Q=[a, b, c]$ a quadratic form of discriminant $d$, define $j(Q)=j\left(\tau_{Q}\right)$ and

$$
f(Q)=\left\{\begin{array}{cl}
\zeta^{b\left(a-c-a c^{2}\right)} \mathfrak{f}\left(\tau_{Q}\right) & \text { if } 2|a, 2| c, \\
\varepsilon_{d} \zeta^{b\left(a-c-a c^{2}\right)} \mathfrak{f}_{1}\left(\tau_{Q}\right) & \text { if } 2 \mid a, 2 \nmid c, \\
\varepsilon_{d} \zeta^{b\left(a-c+a^{2} c\right)} \mathfrak{f}_{2}\left(\tau_{Q}\right) & \text { if } 2 \nmid a, 2 \mid c,
\end{array}\right.
$$

where $\varepsilon_{d}=(-1)^{(d-1) / 8}$ and $\zeta=e^{2 \pi i / 48}$. Then:

(i) $f(Q)$ depends only on the $S L_{2}(\mathbb{Z})$-equivalence class $[Q]$ of $Q$.

(ii) $f(Q) \in \mathbb{Q}(j(Q))$.

By (i), we can write $f([Q])$ for $f(Q)$. We call the $h(d)$ numbers $f(\mathcal{A}), \mathcal{A} \in$ $\operatorname{Pic}(\mathcal{O})$, the Weber singular moduli or Weber class invariants for the discriminant d. The minimal polynomial of $f\left(\mathcal{A}_{0}\right)$, where $\mathcal{A}_{0} \in \operatorname{Pic}(\mathcal{O})$ is the principal class (corresponding to the form $Q_{0}=[1,1,(1-d) / 4]$ ), will be denoted $W_{d}(X)$ and called the Weber polynomial for the discriminant $d$. It follows from (ii) of the proposition that the roots of $W_{d}(X)$, i.e. the conjugates of $f\left(\mathcal{A}_{0}\right)$, are up to sign the numbers $f(\mathcal{A})$. Indeed, we know that each $j(Q)$ is a conjugate of $j\left(Q_{0}\right)$, so the corresponding conjugate of $f(Q)$ is a real root of the equation $\left(X^{24}-16\right)^{3}=j\left(Q_{0}\right) X^{24}$. But this 
equation has only two real roots $\pm f\left(Q_{0}\right)$ (since as a cubic in $X^{24}$ it has negative discriminant and hence only one real root, and a real number has only two real 24th roots). The study of a large number of examples suggests that the $f(\mathcal{A})$ themselves, and not merely their squares, are conjugates of one another (the choice of sign in the definition of $f(Q)$ was based on these examples). The truth of this conjecture could be verified by carefully working out the statement of the Shimura reciprocity law in this situation, but we have not done this. If it is true, then we have

$$
W_{d}(X)=\prod_{\mathcal{A} \in \operatorname{Pic}(\mathcal{O})}(X-f(\mathcal{A})) .
$$

Proof of the Proposition. To prove (i), we must check the invariance under the generators of $S L_{2}(\mathbb{Z})$. Let $Q=[a, b, c], Q^{*}=[c,-b, a]$, so that $\tau_{Q^{*}}=-1 / \tau_{Q}$. Then, using the transformation formulas for the Weber functions under $\tau \mapsto-1 / \tau$, we find

$$
f\left(Q^{*}\right)=\left\{\begin{array}{cl}
\zeta^{-b\left(c-a-a^{2} c\right)} \mathfrak{f}\left(\tau_{Q}\right) & \text { if } 2|a, 2| c, \\
\varepsilon_{d} \zeta^{-b\left(c-a+a c^{2}\right)} \mathfrak{f}_{1}\left(\tau_{Q}\right) & \text { if } 2 \mid a, 2 \nmid c, \\
\varepsilon_{d} \zeta^{-b\left(c-a-a^{2} c\right)} \mathfrak{f}_{2}\left(\tau_{Q}\right) & \text { if } 2 \nmid a, 2 \mid c,
\end{array}\right.
$$

so $f\left(Q^{*}\right)$ and $f(Q)$ are the same if $a$ or $c$ is odd and differ by a factor $\zeta^{a b c(a+c)}$ if both are even. But in the latter case $a b c(a+c)$ is always divisible by $48(a / 2, c / 2$ and $(a+c) / 2$ cannot all be odd, and $b^{2}-4 a c \not \equiv 0(\bmod 3)$ implies that at least one of $a, b, c, a+c$ is divisible by 3 ). Similarly, if we take $Q^{*}=[a, b-2 a, c-b+a]$, with $\tau_{Q^{*}}=\tau_{Q}+1$, then using the transformation formulas for the Weber functions under $\tau \mapsto \tau+1$ we find that the desired equality $f\left(Q^{*}\right)=f(Q)$ is equivalent to the congruences

$$
\begin{array}{rll}
(b-2 a)\left(b-c-a(c-b+a)^{2}\right)-1+3(d-1) \equiv b\left(a-c-a c^{2}\right) & (\bmod 48) & \text { if } 2 \mid a, \\
(b-2 a)\left(b-c+a^{2}(c-b+a)\right)+2 \equiv b\left(a-c+a^{2} c\right) & (\bmod 48) & \text { if } 2 \nmid a,
\end{array}
$$

and these can be checked by case-by-case analysis. This proves (i).

Now the $S L_{2}(\mathbb{Z})$-invariance lets us give an alternate definition of $f(\mathcal{A})$ : choose a representative $Q=[a, b, c]$ for $\mathcal{A}$ with $(a, 6)=1$ (this is possible because a primitive form represents numbers prime to any fixed modulus) and with $b \equiv-a(\bmod 24)$ (this can be done by changing $b$ by a suitable multiple of $2 a$, since $b$ is odd). Then since $a^{2} \equiv 1(\bmod 24)$ we find

$$
f(\mathcal{A})=\varepsilon_{d} \zeta^{-1} \mathfrak{f}_{2}\left(\tau_{Q}\right)=\varepsilon_{d} \sqrt{2} / \mathfrak{f}\left(2 \tau_{Q}-1\right),
$$

where we have used the identity $\mathfrak{f}_{2}(\tau)=\zeta \sqrt{2} / \mathfrak{f}(2 \tau-1)$. But $\varpi=2 \tau_{Q}-1$ is a root of the quadratic equation $A \varpi^{2}+2 B \varpi+C=0$ with $A(=a)$ and $C(=a+b+c)$ both odd, $B(=a+b)$ divisible by 24 , and $A C-B^{2}(=|d|)$ congruent to 7 modulo 8 and to \pm 1 modulo 3 , and for such a number Weber showed that $\sqrt{2} \mathfrak{f}(\varpi)$ is in the same field as the $j$-invariant ([11], $§ 127$; see also [3], [9] for more modern discussions). This proves (ii).

\section{EXAmples of Weber Class EqUations, THEIR DisCRiminants AND RESULTANTS}

As we just saw, we could have defined the Weber invariants $f(\mathcal{A})$ as $\varepsilon_{d} \sqrt{2} / \mathfrak{f}\left(2 \tau_{Q}-1\right)$, where $Q=[a, b, c]$ is any form in $\mathcal{A}$ with $(a, 6)=1$ and $24 \mid a+b$, rather than by the more complicated formulas in the proposition. The advantage of 
TABLE 1. Some Weber class polynomials and their discriminants

\begin{tabular}{|c|c|c|c|}
\hline$|d|$ & $h(d)$ & $W_{d}(x)$ & $\operatorname{Disc}\left(W_{d}\right)$ \\
\hline 7 & 1 & 1,1 & 1 \\
\hline 23 & 3 & $1,-1,0,1$ & -23 \\
\hline 31 & 3 & $1,0,1,-1$ & -31 \\
\hline 47 & 5 & $1,2,2,1,0,-1$ & $47^{2}$ \\
\hline 55 & 4 & $1,1,0,-2,-1$ & $-5^{2} 11$ \\
\hline 71 & 7 & $1,-1,-1,1,-1,-1,2,1$ & $-71^{3}$ \\
\hline 79 & 5 & $1,-1,1,-2,3,-1$ & $79^{2}$ \\
\hline 95 & 8 & $1,-1,0,1,-2,-1,2,2,-1$ & $-5^{4} 17^{2} 19^{3}$ \\
\hline 103 & 5 & $1,-2,3,-3,1,1$ & $103^{2}$ \\
\hline 119 & 10 & $1,1,2,4,5,7,9,8,5,4,1$ & $7^{4} 17^{5} 19^{2}$ \\
\hline 127 & 5 & $1,-1,-2,1,3,-1$ & $127^{2}$ \\
\hline 143 & 10 & $1,-3,6,-6,3,3,-9,13,-12,6,-1$ & $5^{2} 11^{4} 13^{5}$ \\
\hline 167 & 11 & $1,-1,5,-4,10,-6,11,-7,9,-4,2,1$ & $-17^{2} 167^{5}$ \\
\hline 175 & 6 & $1,1,0,0,0,-4,1$ & $3^{4} 5^{5} 7^{2}$ \\
\hline 191 & 13 & $1,-2,0,4,-5,1,5,-11,19,-22,16,-10,6,-1$ & $7^{4} 41^{2} 191^{6}$ \\
\hline 215 & 14 & $1,2,0,-6,-3,8,13,-4,-16,-7,13,11,-4,-6,-1$ & $5^{11} 13^{2} 19^{2} 43^{6}$ \\
\hline 239 & 15 & $1,-4,4,4,-5,-13,20,4,-15,-13,27,-4,-8,-2,6,-1$ & $-13^{2} 19^{2} 139^{2} 239^{7}$ \\
\hline 247 & 6 & $1,-3,6,-7,7,-4,-1$ & $3^{2} 13^{3} 19^{2}$ \\
\hline 311 & 19 & $\begin{array}{l}1,-1,2,-5,8,-14,13,-10,-1,9,-18,25,-10,-4,38 \\
\quad-42,37,-16,4,1\end{array}$ & $\begin{array}{c}-17^{4} 19^{2} 23^{4} 103^{2} \\
\cdot 211^{2} 311^{9}\end{array}$ \\
\hline 319 & 10 & $1,5,11,14,10,2,1,5,9,6,-1$ & $3^{8} 11^{4} 29^{5}$ \\
\hline 335 & 18 & $\begin{array}{l}1,5,14,25,33,42,64,102,144,171,179,174,163,144,106, \\
\quad 55,20,4,-1\end{array}$ & $\begin{array}{l}5^{9} 11^{2} 17^{2} 41^{2} 67^{8} 73^{2} \\
\quad \cdot 127^{2} 139^{2}\end{array}$ \\
\hline 367 & 9 & $1,-2,-1,6,-2,0,2,-3,9,-1$ & $3^{6} 19^{2} 367^{4}$ \\
\hline 383 & 17 & $1,-1,-1,-1,0,1,13,7,11,4,1,7,23,31,42,24,6,-1$ & $5^{6} 11^{4} 13^{6} 59^{2} 89^{2} 383^{8}$ \\
\hline 407 & 16 & $1,-1,2,-1,9,2,15,0,12,0,4,-19,-17,-33,-4,-10,-1$ & $-5^{4} 11^{7} 37^{8} 41^{4} 83^{2} 97^{2} 199^{2}$ \\
\hline 431 & 21 & $\begin{array}{l}1,3,6,9,9,-4,-10,-36,-30,-14,-2,66,41,83,44 \\
\quad 10,21,-40,16,-15,12,-1\end{array}$ & $\begin{array}{l}13^{10} 31^{2} 43^{2} 73^{2} 107^{2} \\
\quad \cdot 331^{2} 431^{10}\end{array}$ \\
\hline 455 & 20 & $\begin{array}{l}1,-6,15,-23,26,-16,-15,57,-73,30,76,-194,246 \\
\quad-191,38,129,-200,142,-50,6,1\end{array}$ & $\begin{array}{l}5^{14} 7^{14} 11^{2} 13^{14} 43^{2} 73^{2} \\
\quad .97^{2} 131^{2}\end{array}$ \\
\hline 479 & 25 & $\begin{array}{c}1,3,9,22,41,60,66,47,6,-48,-82,-76,11,138,280 \\
336,317,205,144,109,126,104,76,23,10,-1\end{array}$ & $\begin{array}{l}13^{6} 17^{6} 31^{6} 41^{4} 79^{2} 113^{2} \\
\quad \cdot 283^{2} 379^{2} 479^{12}\end{array}$ \\
\hline
\end{tabular}

the definition we gave is that $f(\mathcal{A})$ can be computed using the root of any representative $Q$, so we can assume $Q$ is reduced $(|b| \leq a \leq c)$. Then $\tau_{Q}$ lies in the standard fundamental domain of $S L_{2}(\mathbb{Z})$ and the Weber functions converge at worst like power series in $e^{-\pi \sqrt{3}} \approx 0.004$, so that a few terms of the product expansion give $f(Q)$ as a complex number to high accuracy. Then $W_{d}(X)$ can be computed using $(2$ ?), where the roots $f(\mathcal{A})$ have been computed to sufficient accuracy to recognize each coefficient of $W_{d}$ as a rational integer. This is much faster than the method used in [8] of computing only one real root to high accuracy and then finding its minimal polynomial by the "LLL" algorithm. Similarly, to compute the discriminant of a Weber class polynomial $W_{d}$, or the resultant of two such polynomials $W_{d_{1}}$ and $W_{d_{2}}$, we compute the product of the differences of the roots as complex numbers to sufficient precision and then round to the nearest integer. The fact that we do in fact get integers to high accuracy provides at the same time a verification of the validity of formula $(2$ ?).

We calculated the polynomials $W_{d}$ and their discriminants for all $d$ satisfying (1) with $-2000<d<0$, obtaining the numerical data which provides the basis for the conjectural formulas given in $\S \S 3-5$. The results for a few values of $d$ in the range $-500<d<0$ are given in Table 1 . In this table, we have given just $h(d)$ and the 
TABLE 2. Resultants of Weber class polynomials

\begin{tabular}{rrrrl}
$\left|d_{1}\right|$ & $\left|d_{2}\right|$ & $h\left(d_{1}\right)$ & $h\left(d_{2}\right)$ & \multicolumn{1}{l}{ Resultant $\left(W_{d_{1}}, W_{d_{2}}\right) \mid$} \\
\hline 71 & 119 & 7 & 10 & $23^{2} 523$ \\
151 & 295 & 7 & 8 & $3^{8} 179 \cdot 211$ \\
119 & 215 & 10 & 14 & $19^{5} 23^{4} 1531$ \\
143 & 239 & 7 & 15 & $13 \cdot 43^{2} 107 \cdot 139^{2} 2131$ \\
167 & 335 & 11 & 18 & $5^{4} 17^{6} 79 \cdot 331 \cdot 379 \cdot 499 \cdot 739$ \\
$* 175$ & 239 & 6 & 15 & $19^{2} 41 \cdot 59^{2} 2411$ \\
191 & 407 & 13 & 16 & $7^{4} 11 \cdot 31^{2} 41^{4} 73 \cdot 83 \cdot 4003 \cdot 4507$ \\
215 & 479 & 14 & 25 & $13^{6} 17^{2} 19^{4} 29^{4} 47^{2} 691 \cdot 877^{2} 1051 \cdot 2179$ \\
247 & 431 & 6 & 21 & $7^{4} 599 \cdot 1427 \cdot 2963 \cdot 6491$ \\
287 & 367 & 14 & 9 & $5^{7} 29^{2} 263^{2} 5003$ \\
311 & 191 & 19 & 13 & $11^{4} 19^{3} 29^{2} 31^{5} 71^{2} 2011$ \\
319 & 383 & 10 & 17 & $11^{4} 13^{6} 163^{2} 1847 \cdot 6947$ \\
$* 343$ & 431 & 7 & 21 & $13^{2} 131 \cdot 181^{2} 251 \cdot 3947 \cdot 8627 \cdot 8699$ \\
407 & 287 & 16 & 14 & $5^{6} 7^{4} 139 \cdot 149^{2} 179^{2} 787 \cdot 811 \cdot 1231$ \\
431 & 263 & 21 & 13 & $7^{12} 167 \cdot 257^{2} 409 \cdot 617^{2} 787 \cdot 883$ \\
455 & 431 & 20 & 21 & $7^{4} 47^{2} 71^{2} 113^{2} 131 \cdot 181 \cdot 199 \cdot 239^{2} 251 \cdot 359^{2} 1291 \cdot 1699 \cdot 2111^{2} 12211$ \\
479 & 359 & 25 & 19 & $13^{6} 19^{2} 31 \cdot 43^{3} 67^{3} 113^{2} 157 \cdot 227 \cdot 239^{2} 761^{2} 977^{2} 1163$ \\
\hline 55 & 95 & 4 & 8 & $23^{2}$ \\
$* 175$ & 287 & 6 & 14 & $5 \cdot 59^{2} 419 \cdot 2099$ \\
247 & 455 & 6 & 20 & $5^{2} 11^{2} 47^{2} 4931 \cdot 7019$ \\
287 & $* 343$ & 14 & 7 & $5^{5} 31^{2} 59 \cdot 61^{2} 4451$ \\
319 & 407 & 10 & 16 & $7^{8} 89^{2} 113^{2} 1151 \cdot 3659$
\end{tabular}

coefficients of $X^{i}$ in $W_{d}(X)(h(d) \geq i \geq 0)$ instead of writing out all the powers in $W_{d}(X)$; thus the fifth entry of the table means that $W_{-55}(X)$ is $X^{4}+X^{3}-2 X-1$, with discriminant $-275=-5^{2} 11$.

For the moment we observe only that the primes dividing the discriminant of $W_{d}(X)$ in Table 1 are always $\leq|d|$, with the power of $|d|$ (if $|d|$ is prime) being $(h(d)-1) / 2$; in $\S 5$ we will make a more detailed analysis of the data and give a precise conjecture about the prime decompositions. The sign of the discriminant is easily determined and not particularly interesting (for instance, it is positive if $h(d) \equiv 1$ or 2 modulo 4 and negative if $h(d) \equiv 3$ modulo 4 ).

In Table 2 we give the resultant of $W_{d_{1}}$ and $W_{d_{2}}$ for various pairs of discriminants $d_{1}, d_{2}$. The last five entries, marked off by a line, give some examples where $\left(d_{1}, d_{2}\right) \neq 1$, while the discriminants marked with an asterisk are not fundamental. We give only the absolute values since the sign of the resultant is easily computable and not very interesting (for instance, if $d_{1}$ and $d_{2}$ are prime, then the sign depends only on $d_{1}$ and $d_{2}$ modulo 16 if $d_{1} \equiv d_{2}+8(\bmod 16)$ and on $\operatorname{sgn}\left(d_{1}-d_{2}\right)$ if $d_{1} \equiv d_{2}$ $(\bmod 16))$.

This time we make the following observations: First, the primes dividing the resultant of $W_{d_{1}}$ and $W_{d_{2}}$ are bounded by $d_{1} d_{2} / 16$. Looking more carefully, we find that if $p$ is such a prime, then $p$ divides some positive integer $m$ of the form $\left(d_{1} d_{2}-x^{2}\right) / 16$ satisfying $m \not \equiv 1(\bmod 3)$ and either $m \equiv 7(\bmod 8)$ or $m \equiv 4$ $(\bmod 16)$ or $m \equiv 0(\bmod 32)$. Refinements of these observations will lead in $\S 3$ to a complete conjectural formula for the resultants.

\section{The PRIMe FACTORIZATION OF THE RESUltants}

Let $Q_{1}$ and $Q_{2}$ be quadratic forms of (the same or different) discriminants $d_{1}$ and $d_{2}$ satisfying (1). Because of the relation $j(Q)=\left(f(Q)^{24}-16\right)^{3} / f(Q)^{24}$, we see 
that any prime dividing $f\left(Q_{1}\right)-f\left(Q_{2}\right)$ also divides $j\left(Q_{1}\right)-j\left(Q_{2}\right)$. Hence to find the formula for the factorization of the norm of $f\left(Q_{1}\right)-f\left(Q_{2}\right)$, we start with the result of [7] on the factorization of $\mathcal{N}\left(j\left(Q_{1}\right)-j\left(Q_{2}\right)\right)$. This involves an arithmetic function $\mathfrak{F}$ (depending on $d_{1}$ and $d_{2}$ ) whose definition we first recall.

Suppose that $d_{1}$ and $d_{2}$ are fixed negative discriminants. For convenience we exclude the values -3 and -4 (later $d_{1}$ and $d_{2}$ will be assumed to satisfy (1)). We also assume that $d_{1}$ and $d_{2}$ are coprime. Then for a prime $p$ such that $\left(\frac{d_{1} d_{2}}{p}\right) \neq-1$ we define

$$
\varepsilon(p)= \begin{cases}\left(\frac{d_{1}}{p}\right) & \text { if } p \nmid d_{1}, \\ \left(\frac{d_{2}}{p}\right) & \text { if } p \nmid d_{2}\end{cases}
$$

(note that this is well-defined and always equal to \pm 1 ). For a positive integer $n$ all of whose prime factors satisfy $\left(\frac{d_{1} d_{2}}{p}\right) \neq-1$ we extend $\varepsilon$ multiplicatively, i.e., we define $\varepsilon(n)=\prod_{p \mid n} \varepsilon(p)^{\operatorname{ord}_{p}(n)}$, where $\operatorname{ord}_{p}(n)$ denotes the power of $p$ dividing $n$. Finally, if $m$ is such that $\varepsilon(m)$ is defined and equal to -1 , we set

$$
\mathfrak{F}(m)=\prod_{\substack{n n^{\prime}=m \\ n, n^{\prime}>0}} n^{\varepsilon\left(n^{\prime}\right)} .
$$

An easy proposition, proved in [7], is that $\mathfrak{F}(m)$ is always a prime power:

$$
\mathfrak{F}(m)=\ell^{(a+1)\left(b_{1}+1\right) \cdots\left(b_{s}+1\right)}
$$

if $m$ has the form

$$
m=\ell^{2 a+1} p_{1}^{2 a_{1}} \cdots p_{r}^{2 a_{r}} q_{1}^{b_{1}} \cdots q_{s}^{b_{s}} \quad\left(\varepsilon(\ell)=\varepsilon\left(p_{i}\right)=-1, \quad \varepsilon\left(q_{i}\right)=1 \text { for all } i\right)
$$

(i.e., if there is a unique prime $\ell$ with $\varepsilon(\ell)=-1$ and $\operatorname{ord}_{\ell}(m)$ odd) and $\mathfrak{F}(m)=1$ otherwise (i.e., if there are three or more such primes; note that there are always an odd number of them since $\varepsilon(m)=-1$ ). The main result of [7] is the formula

$$
\mathcal{N}\left(j\left(Q_{1}\right)-j\left(Q_{2}\right)\right)^{2}=\prod \mathfrak{F}\left(\frac{d_{1} d_{2}-x^{2}}{4}\right),
$$

where the product is taken over all $x$ such that $x^{2}<d_{1} d_{2}$ and that $x^{2} \equiv d_{1} d_{2}$ $(\bmod 4)$. Note that each of the integers $m=\left(d_{1} d_{2}-x^{2}\right) / 4$ satisfies $\varepsilon(m)=-1$, so that $\mathfrak{F}(m)$ is defined. If $d_{1}$ and $d_{2}$ are both odd, then $x \neq 0$ and the formula can be simplified to

$$
\mathcal{N}\left(j\left(Q_{1}\right)-j\left(Q_{2}\right)\right)= \pm \prod_{\substack{0<x<\sqrt{d_{1} d_{2}} \\ x \text { odd }}} \mathfrak{F}\left(\frac{d_{1} d_{2}-x^{2}}{4}\right)
$$

This formula implies that any prime $p$ dividing the norm has the following properties:

(i) $\left(\frac{d_{1}}{p}\right) \neq 1$ and $\left(\frac{d_{2}}{p}\right) \neq 1$;

(ii) $p$ divides a positive integer of the form $\frac{d_{1} d_{2}-x^{2}}{4}$, and in particular $p<d_{1} d_{2} / 4$;

(iii) $p<d_{1} d_{2} / 8$ if $d_{1}$ and $d_{2}$ are both congruent to 1 modulo 8 .

We now want to find a similar type of formula for the norm of $f\left(Q_{1}\right)-f\left(Q_{2}\right)$, i.e., for the resultant of the Weber polynomials $W_{d_{1}}$ and $W_{d_{2}}$. Since this norm divides 
that of $j\left(Q_{1}\right)-j\left(Q_{2}\right)$, as already mentioned, we know that only primes satisfying (i)-(iii) can occur. Looking at Table 2 in the previous section, we find that this is true and that in fact (iii) can be strengthened to $p<d_{1} d_{2} / 16$. However, even after studying this table (and a much larger collection of numerical results of which it is only an excerpt) in detail it was not obvious what the exact rule was for deciding which primes occurred and to which powers. To find this rule, we first split the problem into a series of simpler ones by passing, not from $j$ directly to $\mathfrak{f}$, which satisfies an algebraic equation of degree 72 over $j$, but first to the cubic extension $\mathbb{C}\left(\mathfrak{f}^{24}\right)$ of $\mathbb{C}(j)$ and then step by step to the $(24 / r)$ th degree extension $\mathbb{C}\left(\mathfrak{f}^{r}\right)$ of this, where $r$ ranges over the divisors of 24 .

The first step is easy. Looking at a number of examples of factorizations of the norm of $f\left(Q_{1}\right)^{24}-f\left(Q_{2}\right)^{24}$, we find that, just as for $f$, the prime divisors are always bounded by $d_{1} d_{2} / 16$ and that the only numbers $m=\left(d_{1} d_{2}-x^{2}\right) / 4$ which contribute prime factors are those divisible by 4 (note that $m$ is always even since $d_{1}$ and $d_{2}$ are 1 modulo 8 and $x$ is even). This suggests the formula

$$
\mathcal{N}\left(f\left(Q_{1}\right)^{24}-f\left(Q_{2}\right)^{24}\right)= \pm \prod_{x>0} \mathfrak{F}\left(\frac{d_{1} d_{2}-x^{2}}{16}\right)
$$

where the product extends over all $x$ between 0 and $\sqrt{d_{1} d_{2}}$ for which $d_{1} d_{2}-x^{2}$ is divisible by 16 , and this formula indeed turns out to be correct experimentally in all cases. To prove it, one would have to repeat the analysis in [7] with $S L_{2}(\mathbb{Z})$ replaced by the group $\Gamma_{0}(2)$.

To go further, we observe that the polynomial $X^{24}-1$ factorizes as $\prod_{r \mid 24} \varphi_{r}(X)$, where $\varphi_{r}(X)$ denotes the $r$ th cyclotomic polynomial. We can write this in the homogeneous form $X^{24}-Y^{24}=\prod_{r \mid 24} \Phi_{r}(X, Y)$ with the $\Phi_{r}(X, Y) \quad\left(r=2^{\alpha} 3^{\beta} \mid 24\right)$ given by the table

\begin{tabular}{|c|c|c|c|c|}
\hline & $\alpha=0$ & $\alpha=1$ & $\alpha=2$ & $\alpha=3$ \\
\hline & $X-Y$ & $X+Y$ & $X^{2}+Y^{2}$ & $X^{4}+Y^{4}$ \\
\hline & $X^{2}+X Y+Y^{2}$ & $X^{2}-X Y+Y^{2}$ & $X^{4}-X^{2} Y^{2}+Y^{4}$ & $X^{8}-X^{4} Y^{4}+Y^{8}$ \\
\hline
\end{tabular}
that

We therefore look for a corresponding decomposition $\mathfrak{F}=\prod_{r \mid 24} \mathfrak{F}_{r}$ in such a way

$$
\mathcal{N}\left(\Phi_{r}\left(f\left(Q_{1}\right), f\left(Q_{2}\right)\right)\right)= \pm \prod_{x>0} \mathfrak{F}_{r}\left(\frac{d_{1} d_{2}-x^{2}}{16}\right) .
$$

To check whether such a formula holds and to see how to define the function $\mathfrak{F}_{r}(m)$ we chose many pairs $\left(d_{1}, d_{2}\right)$ in which some prime $\ell$ divides only one of the numbers $m=\left(d_{1} d_{2}-x^{2}\right) / \ell$ and then looked where $\ell$ appears in the norms of the $\Phi_{r}\left(f\left(Q_{1}\right), f\left(Q_{2}\right)\right)$ 's. To state the result, rewrite equation $(3)$ as $\mathfrak{F}(m)=\ell^{\gamma(m)}$, where $\gamma(m)=\prod_{p \mid m} \gamma_{p}(m)$ with

$$
\gamma_{p}(m)=\left\{\begin{array}{cl}
\operatorname{ord}_{p}(m)+1 & \text { if } \varepsilon(p)=+1 \\
1 & \text { if } \varepsilon(p)=-1, \operatorname{ord}_{p}(m) \text { even, } \\
\frac{1}{2}\left(\operatorname{ord}_{p}(m)+1\right) & \text { if } \left.\varepsilon(p)=-1, \operatorname{ord}_{p}(m) \text { odd (i.e. } p=\ell\right)
\end{array}\right.
$$

(If $\mathfrak{F}(m)=1$, then $\gamma(m)$ is not defined.) 
Conjectural formula for $\mathcal{N}\left(\Phi_{r}\left(f\left(Q_{1}\right), f\left(Q_{2}\right)\right)\right)$. Let $d_{1}$ and $d_{2}$ be coprime fundamental discriminants satisfying (1). Then for each divisor $r$ of 24 the norm of $\Phi_{r}\left(f\left(Q_{1}\right), f\left(Q_{2}\right)\right)$ is given by equation $(6$ ? $)$, where $\mathfrak{F}_{r}(m)$ is defined as 1 if $\mathfrak{F}(m)=1$ and as $\ell^{\gamma(r, m)}$ if $F(m)=\ell^{\gamma(m)}$, with

$$
\gamma(r, m)=\gamma_{2}(\alpha, m) \gamma_{3}(\beta, m) \prod_{p \neq 2,3} \gamma_{p}(m) \quad\left(r=2^{\alpha} 3^{\beta}\right)
$$

and $\gamma_{2}(\alpha, m)$ and $\gamma_{3}(\beta, m)$ defined by the following two tables:

\begin{tabular}{|c|c|c|c|c|}
\hline$\gamma_{2}(\alpha, m):$ & $\alpha=0$ & $\alpha=1$ & $\alpha=2$ & $\alpha=3$ \\
\hline$m \equiv 3(\bmod 8)$ & 1 & 0 & 0 & 0 \\
\hline$m \equiv 7(\bmod 8)$ & 0 & 1 & 0 & 0 \\
\hline$m \equiv 1(\bmod 4)$ & 0 & 0 & 1 & 0 \\
\hline$m \equiv 2(\bmod 4)$ & 0 & 0 & 0 & 2 \\
\hline$m \equiv 12(\bmod 16)$ & 1 & 0 & 0 & 2 \\
\hline$m \equiv 4(\bmod 16)$ & 0 & 1 & 0 & 2 \\
\hline$m \equiv 8(\bmod 16)$ & 0 & 0 & 2 & 2 \\
\hline$m \equiv 16(\bmod 32)$ & 1 & 0 & 2 & 2 \\
\hline$m \equiv 0(\bmod 32)$ & $\operatorname{ord}_{2}(m)-5$ & 2 & 2 & 2 \\
\hline
\end{tabular}

\begin{tabular}{|c|c|c|c|}
\hline \multirow{7}{*}{$\begin{array}{l}\left(\frac{d_{1}}{3}\right)=-\left(\frac{d_{2}}{3}\right), \\
\left(\frac{d_{1}}{3}\right)=-\left(\frac{d_{2}}{3}\right), \\
\left(\frac{d_{2}}{3}\right)=+1, \\
\left(\frac{d_{2}}{3}\right)=+1, \\
\left(\frac{d_{2}}{3}\right)=-1, \\
\left(\frac{d_{2}}{3}\right)=-1,\end{array}$} & & & \multirow{2}{*}{$\beta=1$} \\
\hline & & & \\
\hline & & \multirow{5}{*}{$\begin{array}{c}\beta=0 \\
1 \\
0\end{array}$} & 1 \\
\hline & & & 0 \\
\hline & \multirow{3}{*}{$\begin{array}{l}\gamma_{3}(\beta, m): \\
m \equiv 2(\bmod 3) \\
m \equiv 1(\bmod 3) \\
m \equiv 1(\bmod 3) \\
m \equiv 0(\bmod 3) \\
\operatorname{ord}_{3}(m) \text { even } \\
\operatorname{ord}_{3}(m) \text { odd }\end{array}$} & & 2 \\
\hline & & & 0 \\
\hline & & & $\frac{1}{2}$ \\
\hline
\end{tabular}

Remarks. 1. The resultant of the Weber polynomials $W_{d_{1}}$ and $W_{d_{2}}$, as tabulated in Table 2, corresponds to the case $r=1$ of the conjecture.

2. The main qualitative aspect of the conjecture is that the powers of 2 and of 3 in $r$ depend only on the 2-adic and 3-adic nature of $m$, respectively, but in a fairly complicated way. In order to be reasonably confident of the formula for the 2-adic part we looked at examples with $\operatorname{ord}_{2}(m)$ going up to 11 .

3. It is easily checked that the congruence conditions on $m$ in each of the two tables cover all possibilities. (For the second table one must observe that the numbers $m$ of the form $\left(d_{1} d_{2}-x^{2}\right) / 16$ are always congruent to 1 or 2 modulo 3 if $\left(d_{1} / 3\right) \neq\left(d_{2} / 3\right)$ and to 0 or 1 modulo 3 if $\left(d_{1} / 3\right)=\left(d_{2} / 3\right)$.)

4. By adding the entries in each row of the tables, one checks that in all cases

$$
\sum_{\alpha=0}^{3} \gamma_{2}(\alpha, m)=\gamma_{2}(m), \quad \sum_{\beta=0}^{1} \gamma_{3}(\beta, m)=\gamma_{3}(m) .
$$

Hence $\sum_{r \mid 24} \gamma(r, m)=\gamma(m)$ and $\prod_{r \mid 24} \mathfrak{F}_{r}(m)=\mathfrak{F}(m)$, so that equation (6?) is compatible with equation $(5$ ?).

5. Since the entries in the last column in the table for $\gamma_{2}(\alpha, m)$ are all even, the conjecture implies that the norms of $\Phi_{8}\left(f\left(Q_{1}\right), f\left(Q_{2}\right)\right)$ and $\Phi_{24}\left(f\left(Q_{1}\right), f\left(Q_{2}\right)\right)$ are always perfect squares, which is indeed observed experimentally. 
TABLE 3. Example of a resultant computation

\begin{tabular}{rrccccccccc}
$x$ & $m$ & $\mathfrak{F}_{1}$ & $\mathfrak{F}_{2}$ & $\mathfrak{F}_{4}$ & $\mathfrak{F}_{8}$ & $\mathfrak{F}_{3}$ & $\mathfrak{F}_{6}$ & $\mathfrak{F}_{12}$ & $\mathfrak{F}_{24}$ & $\mathfrak{F}$ \\
\hline 1 & $2^{4} \cdot 3 \cdot \underline{11}$ & 1 & 1 & 1 & 1 & $11^{2}$ & 1 & $11^{4}$ & $11^{4}$ & $11^{10}$ \\
7 & $3 \cdot 5^{2} \cdot \underline{7}$ & 1 & 1 & 1 & 1 & 1 & 1 & $7^{6}$ & 1 & $7^{6}$ \\
9 & $\underline{523}$ & 523 & 1 & 1 & 1 & 1 & 1 & 1 & 1 & 523 \\
15 & $2 \cdot \underline{257}$ & 1 & 1 & 1 & $257^{2}$ & 1 & 1 & 1 & 1 & $257^{2}$ \\
17 & $2 \cdot 3 \cdot 5 \cdot \underline{17}$ & 1 & 1 & 1 & 1 & 1 & 1 & 1 & $17^{8}$ & $17^{8}$ \\
23 & $3^{2} \cdot 5 \cdot \underline{11}$ & 1 & $11^{4}$ & 1 & 1 & 1 & $11^{2}$ & 1 & 1 & $11^{6}$ \\
25 & $3 \cdot 163$ & 1 & 1 & 1 & 1 & 1 & 1 & $163^{2}$ & 1 & $163^{2}$ \\
31 & $2^{2} \cdot 3^{2} \cdot \underline{13}$ & 1 & 13 & 1 & $13^{2}$ & 1 & $13^{2}$ & 1 & $13^{4}$ & $13^{9}$ \\
33 & $2^{2} \cdot 5 \cdot \underline{23}$ & $23^{2}$ & 1 & 1 & $23^{4}$ & 1 & 1 & 1 & 1 & $23^{6}$ \\
39 & $2 \underline{43}$ & 1 & 1 & 433 & 1 & 1 & 1 & 1 & 1 & 433 \\
41 & $3^{2} \cdot \frac{47}{1}$ & 1 & 47 & 1 & 1 & 1 & $47^{2}$ & 1 & 1 & $47^{3}$ \\
47 & $2 \cdot 3 \cdot 5 \cdot \frac{13}{1}$ & 1 & 1 & 1 & 1 & 1 & 1 & 1 & $13^{8}$ & $13^{8}$ \\
49 & $2 \cdot 3^{3} \cdot \underline{7}$ & 1 & 1 & 1 & $7^{4}$ & 1 & 1 & 1 & $7^{4}$ & $7^{8}$ \\
55 & $3 \cdot \underline{113}$ & 1 & 1 & 1 & 1 & $113^{2}$ & 1 & 1 & 1 & $113^{2}$ \\
57 & $5^{2} \cdot \underline{13}$ & 1 & 1 & $13^{3}$ & 1 & 1 & 1 & 1 & 1 & $13^{3}$ \\
63 & $2^{3} \cdot 5 \cdot \underline{7}$ & 1 & 1 & $7^{4}$ & $7^{4}$ & 1 & 1 & 1 & 1 & $7^{8}$ \\
65 & $2^{3} \cdot 3 \cdot \underline{11}$ & 1 & 1 & 1 & 1 & 1 & 1 & $11^{4}$ & $11^{4}$ & $11^{8}$ \\
71 & $3 \cdot \underline{71}$ & 1 & 1 & 1 & 1 & 1 & 1 & $71^{2}$ & 1 & $71^{2}$ \\
73 & $3 \cdot 5 \cdot \frac{13}{13}$ & 1 & 1 & 1 & 1 & $13^{4}$ & 1 & 1 & 1 & $13^{4}$ \\
79 & $2 \cdot 3 \cdot \underline{23}$ & 1 & 1 & 1 & 1 & 1 & 1 & 1 & $23^{4}$ & $23^{4}$ \\
81 & $2 \cdot \underline{59}$ & 1 & 1 & 1 & $59^{2}$ & 1 & 1 & 1 & 1 & $59^{2}$ \\
87 & $5 \cdot \underline{11}$ & 1 & 1 & 1 & 1 & 1 & $11^{2}$ & 1 & 1 & $11^{2}$ \\
89 & $3 \cdot \underline{11}$ & 1 & 1 & 1 & 1 & 1 & 1 & $11^{2}$ & 1 & $11^{2}$
\end{tabular}

6. The entries in the last row of the second table, corresponding to the case when $\ell=3$, are half-integers. Therefore $\gamma(m)$ can be a half-integer in this case. However, this happens if and only if $m$ has the form $3 y^{2}$ for some $y>0$, since the formulas for $\gamma_{p}(m)$ and $\gamma_{2}(\alpha, m)$ imply that these numbers are even if $\operatorname{ord}_{p}(m)\left(\operatorname{resp}_{\operatorname{cod}}(m)\right)$ is odd. Thus $\mathfrak{F}_{r}(m)$ is an integer multiple of $\sqrt{3}$ if $m=3 y^{2}$ and an integer otherwise. Since the number of representations of $d_{1} d_{2}$ as $x^{2}+48 y^{2}$ under our assumptions on $d_{1}$ and $d_{2}$ is always even, the formula stated always yields an integral value for $\mathcal{N}\left(\Phi_{r}\left(f\left(Q_{1}\right), f\left(Q_{2}\right)\right)\right)$, as it should.

Example. Take $d_{1}=-71, d_{2}=-119$, with class numbers 7 and 10, respectively. Table 3 gives the data needed to compute the numbers $\mathcal{N}\left(\Phi_{r}\left(f\left(Q_{1}\right), f\left(Q_{2}\right)\right)\right)$ for each divisor $r$ of 24. The table shows the prime factorizations of the numbers $m=$ $\left(d_{1} d_{2}-x^{2}\right) / 16=\left(8449-x^{2}\right) / 16$, the underlined prime factor being $\ell$, and gives the values of $\mathfrak{F}_{r}(m)(r \mid 24)$ and of their product $\mathfrak{F}(m)$. From the table we read off that the absolute value of the norm of $f\left(Q_{1}\right)-f\left(Q_{2}\right)$ is $23^{2} 523$ (the first entry of Table 2), while that of $f\left(Q_{1}\right)^{24}-f\left(Q_{2}\right)^{24}$ is $7^{22} 11^{28} 13^{24} 17^{8} 23^{10} 47^{3} 59^{2} 71^{2} 113^{2} 163^{2} 257^{2} 433523$ $\left(\approx 8.1 \times 10^{128}\right)$.

\section{Simplification of the formula for the Resultant}

The conjectural formula for the norm of $\Phi_{r}\left(f\left(Q_{1}\right)^{r}-f\left(Q_{2}\right)\right)$ which we gave in the last section is complete and is easy to apply numerically, but has the aesthetic disadvantage that the definition of the crucial function $\mathfrak{F}_{r}(m)$ is given in terms of the 48 entries of two tables which were found experimentally and for which no unified description was given. In this section we will find such a unified description 
TABle 4 . The sets $\Gamma_{2}(\alpha, m)$

\begin{tabular}{|c|c|c|c|c|}
\hline \multirow[b]{2}{*}{$m \equiv 3(\bmod 8)$} & $\alpha=0$ & $\alpha=1$ & $\alpha=2$ & $\alpha=3$ \\
\hline & $\{1\}$ & - & - & - \\
\hline$m \equiv 7(\bmod 8)$ & - & $\{1\}$ & - & - \\
\hline$m \equiv 1(\bmod 4)$ & - & - & $\{1\}$ & - \\
\hline$m \equiv 2(\bmod 4)$ & E- & - & - & $\{\mathfrak{p}, \overline{\mathfrak{p}}\}$ \\
\hline$m \equiv 12(\bmod 16)$ & $\{\mathfrak{p} \overline{\mathfrak{p}}\}$ & - & - & $\left\{\mathfrak{p}^{2}, \overline{\mathfrak{p}}^{2}\right\}$ \\
\hline$m \equiv 4(\bmod 16)$ & - & $\{\mathfrak{p} \overline{\mathfrak{p}}\}$ & - & $\left\{\mathfrak{p}^{2}, \overline{\mathfrak{p}}^{2}\right\}$ \\
\hline$m \equiv 8(\bmod 16)$ & - & - & $\left\{\mathfrak{p}^{2} \overline{\mathfrak{p}}, \mathfrak{p} \overline{\mathfrak{p}}^{2}\right\}$ & $\left\{\mathfrak{p}^{3}, \overline{\mathfrak{p}}^{3}\right\}$ \\
\hline$m \equiv 16(\bmod 32)$ & $\left\{\mathfrak{p}^{2} \overline{\mathfrak{p}}^{2}\right\}$ & - & $\left\{\mathfrak{p}^{3} \overline{\mathfrak{p}}, \mathfrak{p} \overline{\mathfrak{p}}^{3}\right\}$ & $\left\{\mathfrak{p}^{4}, \overline{\mathfrak{p}}^{4}\right\}$ \\
\hline $2^{\nu} \| m, \nu \geq 5$ & $\left\{\mathfrak{p}^{i} \overline{\mathfrak{p}}^{\nu-i}\right\}_{3 \leq i \leq \nu-3}$ & $\left\{\mathfrak{p}^{\nu-2} \overline{\mathfrak{p}}^{2}, \mathfrak{p}^{2} \overline{\mathfrak{p}}^{\nu-2}\right\}$ & $\left\{\mathfrak{p}^{\nu-1} \overline{\mathfrak{p}}, \mathfrak{p} \overline{\mathfrak{p}}^{\nu-1}\right\}$ & $\left\{\mathfrak{p}^{\nu}, \overline{\mathfrak{p}}^{\nu}\right\}$ \\
\hline
\end{tabular}

by a series of successive simplifications; the form we obtain finally will still be a little mysterious, but will involve far fewer "experimental constants."

The formula for the case $r=24$, equation ( 5 ?), was a natural enough analogue of the known equation (4) for the level 1 case; the mystery concerned only the splitting of the arithmetic function $\mathfrak{F}$ as $\prod_{r \mid 24} \mathfrak{F}_{r}$, or equivalently of $\gamma(m)$ as $\sum_{r \mid 24} \gamma(r, m)$, where $\mathfrak{F}(m)=\ell^{\gamma(m)}$. (If $\mathfrak{F}(m)=1$, then there is nothing to discuss.) Suppose that we have a quadratic field $K$ in which every prime $p \mid m$ with $\varepsilon(p)=1$ splits and every prime $p \mid m$ with $\varepsilon(p)=-1$ is inert. The choice of this field is arbitrary and in this section will just be a convenient device for counting ideals; in $\S 5$, where we consider the case $d_{1}=d_{2}$, there will be a canonical choice $K=\mathbb{Q}\left(\sqrt{d_{1}}\right)$. Then $\gamma(m)$ counts the number of ideals in $K$ whose norm has the form $m / \ell^{k}$ for some (necessarily odd) integer $k$ with $\ell^{k} \mid m$, and $\gamma_{p}(m)$ for $p \neq \ell$ counts the number of ideals of $K$ of norm $p^{\operatorname{ord}_{p}(m)}$. Denote this set of ideals by $\Gamma_{p}(m)$, so that $\gamma_{p}(m)=\left|\Gamma_{p}(m)\right|$. Let us try to realize the decompositions $(7)$ of $\gamma_{2}(m)$ and $\gamma_{3}(m)$ by corresponding decompositions $\Gamma_{2}(m)=\bigcup_{\alpha=0}^{3} \Gamma_{2}(\alpha, m), \Gamma_{3}(m)=\bigcup_{\beta=0}^{1} \Gamma_{3}(\beta, m)$.

We start with $p=2$. Since we are assuming that $d_{1} \equiv d_{2} \equiv 1(\bmod 8)$, we have $\varepsilon(2)=+1$ and $(2)=\mathfrak{p} \overline{\mathfrak{p}}$, so $\Gamma_{2}(m)=\left\{\mathfrak{p}^{\nu}, \mathfrak{p}^{\nu-1} \overline{\mathfrak{p}}, \ldots, \overline{\mathfrak{p}}^{\nu}\right\}$, where $2^{\nu} \| m$. We partition this set into four subsets $\Gamma_{2}(\alpha, m)$ according to Table 4 . This is the only "reasonable" way to match the decomposition of $\gamma_{2}(m)$ given by the table in the conjecture. Indeed, for the first four rows there is no choice at all, in the next two rows no other choice which is invariant under the action of $\operatorname{Gal}(K / \mathbb{Q})$, and then the last three rows are the natural continuation of the pattern evident in the first six. (We will give a more objective reason for the choice made here in $\S 5$, where there is a canonical choice of $K$ and a way to distinguish the different ideals.)

An inspection of Table 4 shows that the column (i.e., the value of $\alpha$ ) to which a given ideal $\mathfrak{b}=\mathfrak{p}^{i} \overline{\mathfrak{p}}^{\nu-i} \in \Gamma_{2}(m)$ is assigned depends only on the minimum of $i$ and $\nu-i$, i.e., only on the largest power of 2 which divides $\mathfrak{b}$, and is given simply by:

$$
\begin{aligned}
& 2 \nmid \mathfrak{b} \Rightarrow \alpha=\max \left\{0,3-\operatorname{ord}_{2}(m-3)\right\} \text {, } \\
& 2 \| \mathfrak{b} \Rightarrow \alpha=\max \left\{0,2-\operatorname{ord}_{2}(m / 4-3)\right\} \text {, } \\
& 4 \| \mathfrak{b} \Rightarrow \alpha=\max \left\{0,1-\operatorname{ord}_{2}(m / 16-1)\right\} \text {, } \\
& 8 \mid \mathfrak{b} \Rightarrow \alpha=0 .
\end{aligned}
$$

Now recall that $\gamma(m)$ counts the number of decompositions as

$$
m=\ell^{k} \mathcal{N}(\mathfrak{a}) \quad(k \geq 1 \text { odd, } \mathfrak{a} \text { an integral ideal of } K)
$$


and that the ideal $\mathfrak{b}$ represents the 2 -primary part of the ideal $\mathfrak{a}$. Denote by $c$ the content of $\mathfrak{a}$, i.e., the largest rational integer such that $\mathfrak{a}=c \mathfrak{a}_{0}$ for some integral ideal $\mathfrak{a}_{0}$. Then we can write the formula just obtained in a uniform way as

$$
2^{\alpha}=\frac{8 /(8, c)}{\left(8 /(8, c), m /(8, c)^{2}-3\right)}
$$

where $($,$) denotes greatest common divisor. To simplify this even further, write m$ as $m_{0} c^{2}$ (so $m_{0}=\ell^{k} \mathcal{N}\left(\mathfrak{a}_{0}\right)$ ) and observe that $m /(8, c)^{2}$ is congruent to $m_{0}$ modulo $8 /(8, c)$, because $c /(8, c)$ is prime to $8 /(8, c)$ and any number prime to a divisor of 8 has square congruent to 1 modulo this divisor. (This property of 8 is shared by 3 and 24 and will be used again for them.) Hence the entire content of Table 3 can be summarized by saying that we assign to each decomposition (8) the number $\alpha \in\{0,1,2,3\}$ defined by

$$
2^{3-\alpha}=\left(8, c\left(m_{0}-3\right)\right)
$$

We now turn to the prime $p=3$. This is much simpler, except that we have different cases depending on the values of $d_{1}$ and $d_{2}$ modulo 3 . (For the prime 2 this case distinction did not occur since we assumed that both discriminants are 1 modulo 8.) If we assume that $d_{1} \equiv d_{2} \equiv 1(\bmod 3)$, corresponding to the third and fourth rows of the second table in our conjecture, then a discussion exactly like the one for $p=2$, but very much simpler, tells us that we must assign to the decomposition (8) the invariant $\beta \in\{0,1\}$ defined by

$$
3^{1-\beta}=\left(3, c\left(m_{0}-1\right)\right) \quad \text { if } d_{1} \equiv d_{2} \equiv 1 \quad(\bmod 3) ;
$$

and this can then be combined with (9) into the single congruence

$$
\frac{24}{r}=\left(24, c\left(m_{0}+5\right)\right) \quad \text { if } d_{1} \equiv d_{2} \equiv 1 \quad(\bmod 24)
$$

for the number $r=2^{\alpha} 3^{\beta}$. If $d_{1}$ and $d_{2}$ have opposite values modulo 3 , then a similar analysis using the first and second rows of the table for $\gamma_{3}(\beta, m)$ (but even easier, since now $\operatorname{ord}_{p}(m)$ is always 0$)$ gives $3^{1-\beta}=(3, m+1)=\left(3, c_{0}(m+1)\right)$, i.e.

(11) $\frac{24}{r}=\left(24, c\left(m_{0}-11\right)\right) \quad$ if $d_{1} \equiv d_{2} \equiv 1 \quad(\bmod 8), d_{1} d_{2} \equiv 2 \quad(\bmod 3)$.

Finally, if $d_{1} \equiv d_{2} \equiv 2(\bmod 3)$, then we can interpret the last two lines in the table for $\gamma_{3}(\beta, m)$ as saying that we must assign to the decomposition (8) the value $\beta=0$ in all cases except when $\ell=3$ and $\mathcal{N}(\mathfrak{a})$ is prime to 3 , in which case this decomposition is to be counted for both $\beta=0$ and $\beta=1$, with multiplicity $1 / 2$ each.

The formulas we have obtained become even simpler if we go back to the numbers $f\left(Q_{1}\right)^{r}-f\left(Q_{2}\right)^{r}$ rather than $\Phi_{r}\left(f\left(Q_{1}\right), f\left(Q_{2}\right)\right)$. Indeed, since $X^{r}-Y^{r}=$ $\prod_{d \mid r} \Phi_{d}(X, Y)$ we see that the norms of these numbers are (conjecturally) given by a formula like $\left(6_{\text {? }}\right)$ but with the function $\mathfrak{F}_{r}(m)$ replaced by $\prod_{d \mid r} \mathfrak{F}_{d}(m)$. Restricting for simplicity to the case $d_{1} \equiv d_{2} \equiv 1(\bmod 24)$, and observing that for $r \mid 24$ the condition that $\left(24, c\left(m_{0}+5\right)\right)=24 / d$ for some divisor $d$ of $r$ is equivalent to $c\left(m_{0}+5\right) \equiv 0(\bmod 24 / r)$, we get:

Conjectural formula for $\mathcal{N}\left(f\left(Q_{1}\right)^{r}-f\left(Q_{2}\right)^{r}\right)$. Let $d_{1}$ and $d_{2}$ be coprime fundamental discriminants congruent to 1 modulo 24. Pick an imaginary quadratic field $K$ in which every prime $p<\frac{d_{1} d_{2}}{16}$ such that $\left(\frac{d_{1}}{p}\right)+\left(\frac{d_{2}}{p}\right)$ is positive (resp. negative) 
splits (resp. is inert). Then for any $r \mid 24$ and any prime $\ell$, the power of $\ell$ dividing $\mathcal{N}\left(f\left(Q_{1}\right)^{r}-f\left(Q_{2}\right)^{r}\right)$ is equal to the number of decompositions

$$
d_{1} d_{2}=x^{2}+16 \ell^{k} \mathcal{N}(\mathfrak{a})
$$

with $x \geq 1, k \geq 1$, and $\mathfrak{a}$ an integral ideal of $K$ satisfying

$$
c(\mathfrak{a})\left(\ell \frac{\mathcal{N}(\mathfrak{a})}{c(\mathfrak{a})^{2}}+5\right) \equiv 0 \quad\left(\bmod \frac{24}{r}\right)
$$

where $c(\mathfrak{a})$ is the content of $\mathfrak{a}$.

This formulation of the conjecture of $\S 3$ still has the disadvantage that it involves the noncanonical choice of the auxiliary quadratic field $K$, of which no interpretation is given. By using the relationship between the number of representations of an integer as the norm of an integral and as the norm of a primitive integral ideal of $K$, and a kind of Möbius inversion, we can rewrite the whole formula in a way only using the arithmetic function $\mathfrak{F}(m)$. The result of the calculation (again only for $\left.d_{1} \equiv d_{2} \equiv 1(\bmod 24)\right)$ is

$$
\mathcal{N}\left(f\left(Q_{1}\right)^{24 / s}-f\left(Q_{2}\right)^{24 / s}\right)= \pm \prod_{\substack{m, x>0, t \mid s \\ x^{2}+16 m t^{2}=d_{1} d_{2} \\ m \equiv 19 \quad(\bmod s / t)}} \mathfrak{F}(m) .
$$

\section{FACTORIZATions of Discriminants of WeBer Polynomials}

In this section we discuss the case $d_{1}=d_{2}$, so that we are concerned with the discriminant of a single Weber polynomial rather than with the resultant of two different ones. The main ideas which will be needed are already contained in $\S 4$, but are more natural here because we can simply take $\mathbb{Q}\left(\sqrt{d_{1}}\right)$ as our auxiliary quadratic field $K$. For simplicity we will assume that $d_{1}=d_{2}=-p$ is a prime with $-p \equiv 1(\bmod 24)$. Then the class number $h=h(-p)$ of $K=\mathbb{Q}(\sqrt{-p})$ is odd, and we write the corresponding class group as $\operatorname{Pic}\left(\mathcal{O}_{K}\right)=\left\{\mathcal{A}_{0}, \mathcal{A}_{1}^{ \pm 1}, \ldots, \mathcal{A}_{(h-1) / 2}^{ \pm 1}\right\}$ with $\mathcal{A}_{0}$ the principal class.

We start with some preliminary comments which apply equally to each of the modular functions $g(\tau)=j(\tau)$ or $\mathfrak{f}(\tau)^{r}, r \mid 24$. We have $h$ numbers $g(\mathcal{A})(\mathcal{A} \in$ $\operatorname{Pic}\left(\mathcal{O}_{K}\right)$ ) which lie in the Hilbert class field $H$ and are the roots of an irreducible monic polynomial $G(X)=G(g ; X) \in \mathbb{Z}[X]$ and of $K\left(\right.$ so $G(j, X)=H_{d}(X)$ and $\left.G(f, X)=W_{d}(X)\right)$. Because $H / K$ is Galois and abelian, we have

$$
g(\mathcal{A B})-g(\mathcal{B})=\sigma_{\mathcal{B}}\left(g(\mathcal{A})-g\left(\mathcal{A}_{0}\right)\right) \quad\left(\mathcal{A}, \mathcal{B} \in \operatorname{Pic}\left(\mathcal{O}_{K}\right), \quad \mathcal{A} \neq \mathcal{A}_{0}\right)
$$

(here $\mathcal{B} \mapsto \sigma_{\mathcal{B}}$ is the $\operatorname{Artin} \operatorname{map} \operatorname{Pic}\left(\mathcal{O}_{K}\right) \cong \operatorname{Gal}(H / K)$ ), so the discriminant of $G$ factors:

$$
\operatorname{disc}(G)= \pm \prod_{\substack{\mathcal{A}_{1}, \mathcal{A}_{2} \in \operatorname{Pic}\left(\mathcal{O}_{K}\right) \\ \mathcal{A}_{1} \neq \mathcal{A}_{2}}}\left(g\left(\mathcal{A}_{1}\right)-g\left(\mathcal{A}_{2}\right)\right)= \pm \prod_{\substack{\mathcal{A} \in \operatorname{Pic}\left(\mathcal{O}_{K}\right) \\ \mathcal{A} \neq \mathcal{A}_{0}}} \mathcal{N}_{H / K}\left(g(\mathcal{A})-g\left(\mathcal{A}_{0}\right)\right)
$$

Also, it is easy to show that for each $\mathcal{A} \neq \mathcal{A}_{0}$ we have $\mathcal{N}_{H / K}\left(g(\mathcal{A})-g\left(\mathcal{A}_{0}\right)\right)=$ $\pm I(\mathcal{A}) \sqrt{-p}$ for some positive rational integer $I(\mathcal{A})=I(g ; \mathcal{A})$, so finally

$$
\operatorname{disc}(G)= \pm I^{2} p^{(h-1) / 2}, \quad I=\prod_{i=1}^{\frac{1}{2}(h-1)} I\left(\mathcal{A}_{i}\right) .
$$


The number $I$, of course, has a natural interpretation as the index of the order $\mathbb{Z}\left[g\left(\mathcal{A}_{0}\right)\right]$ in the full ring of integers of the maximal real subfield $H^{+}=\mathbb{Q}\left(g\left(\mathcal{A}_{0}\right)\right)$ of $H$. The fact that it decomposes naturally into $(h-1) / 2$ factors means that we have replaced our original problem of determining the discriminant of $G$ by the more refined problem of calculating each factor $I\left(\mathcal{A}_{i}\right)$. We first describe the solution of this problem for the case $g=j$, since this solution was proved but not written out explicitly in [7].

The main result of [7], given here as equations (4) and (8), says that the power of a prime $\ell$ dividing the norm of $j\left(Q_{1}\right)-j\left(Q_{2}\right)$ in the case $d_{1} \neq d_{2}$ is the number of representations of $d_{1} d_{2}$ as $x^{2}+4 \ell^{k} \mathcal{N}(\mathfrak{b})$ with $x, k>0$ and $\mathfrak{b}$ primitive in $K(K$ now defined as in $\S 4$ ); the conjecture in $\S 4$ then says that the same is true for $\mathfrak{f}^{24}$ instead of $j$ if we add the requirement $2 \mid \mathfrak{b}$ (so $\mathfrak{b}=2 \mathfrak{a}$ with $\mathfrak{a}$ satisfying (12)) and for $\mathfrak{f}^{r}$ if we add the congruence condition (13). If we now set $d_{1}=d_{2}=-p$ (and $K=\mathbb{Q}(\sqrt{-p}))$, then the expression $\frac{1}{4}\left(d_{1} d_{2}-x^{2}\right)$ factors as $\frac{1}{2}(p-x) \cdot \frac{1}{2}(p+x)$. Since the two factors are coprime, we find that $\ell^{k}$ divides one factor $\frac{1}{2}(p \pm x)$. Then $\frac{1}{2}(p \pm x)=\ell^{k} \mathcal{N}\left(\mathfrak{b}_{1}\right), \frac{1}{2}(p \mp x)=\mathcal{N}\left(\mathfrak{b}_{2}\right)$ for uniquely determined ideals $\mathfrak{b}_{1}$ and $\mathfrak{b}_{2}$ with $\mathfrak{b}_{1} \mathfrak{b}_{2}=\mathfrak{b}$, so we have to count the representations of $p$ in the form

$$
p=\ell^{k} \mathcal{N}\left(\mathfrak{b}_{1}\right)+\mathcal{N}\left(\mathfrak{b}_{2}\right) \quad\left(k \geq 1, \mathfrak{b}_{1} \text { and } \mathfrak{b}_{2} \text { integral ideals of } K\right) .
$$

The result of [7] was then that the power of $\ell$ in each factor $I\left(j ; \mathcal{A}_{i}\right)$ in $(15)$ is equal to the number of decompositions (16) with $\mathfrak{b}_{2} \in \mathcal{A}_{i}$. (This is proved in Theorem 4.7 of [7] and the three following sentences, but the subsequent Corollary 4.8 gives only the result for the product $I$.) Now going back to our case, it is clear that the logical conjecture is:

Conjectural formula for the discriminant factors. Let $K=\mathbb{Q}(\sqrt{-p})$ with $p \equiv 23(\bmod 24)$ and other notations as above. Then for any prime $\ell, r \mid 24$, and nonprincipal ideal class $\mathcal{A} \in \operatorname{Pic}\left(\mathcal{O}_{K}\right)$, the power of $\ell$ dividing $I\left(f^{r} ; \mathcal{A}\right)$ is equal to the number of representations of $p$ in the form (16) with $\mathfrak{b}_{1}$ and $\mathfrak{b}_{2}$ integral ideals of $K$ with $\mathfrak{b}_{2} \in \mathcal{A}$ and $\mathfrak{b}_{1} \mathfrak{b}_{2}=2 \mathfrak{a}$ for some integral ideal $\mathfrak{a}$ and satisfying (13).

Remarks. 1. If we take $r=1$ and multiply all of the $I(\mathcal{A})$, the conjecture says that the power of a prime $\ell \neq p$ dividing the discriminant of the Weber polynomial $W_{-p}(X)$ equals the number of representations (16) with $\mathfrak{b}_{2}$ nonprincipal and $\mathfrak{a}=$ $\mathfrak{b}_{1} \mathfrak{b}_{2} / 2$ an integral ideal satisfying (13) with $r=1$. This can be tested on the examples in Table 1.

2. When we chose the partition $\Gamma_{2}(m)=\bigcup_{\alpha=0}^{3} \Gamma_{2}(\alpha, m)$ in the way described by Table 4 , the only justification we could give was that this was the most natural way available and led to the simple final formula (9). However, in the case $d_{1}=d_{2}$ the fact that the discriminant factors as in (15) means that we can uniquely recognize the "right" way to partition the set $\Gamma_{2}(m)$ (and similarly $\Gamma_{3}(m)$ ) by looking at the ideal classes to which the ideals in the various subsets must belong to make the formula work out. In fact Table 4 was found in this way, by looking at the various factors of the discriminant of $G\left(f^{r} ; X\right)$ for a large number of quadratic fields and determining the unique way of choosing the entries in the table which was compatible with the numerical data.

Example. We illustrate the conjecture and remarks by one example. Take $p=47$, with class number 5 . Here the discriminant of the Weber polynomial $W_{-p}(X)=$ $G(f, X)$ is uninteresting (compare Table 1 ), but the polynomials $G(j ; X)$ and 
$G\left(f^{r} ; X\right)$ for $r>1$ have nontrivial discriminants. For instance, the polynomial $G(j, X)$ is

$$
\begin{gathered}
x^{5}+2257834125 x^{4}-9987963828125 x^{3}+5115161850595703125 x^{2} \\
-14982472850828613281250 x+16042929600623870849609375
\end{gathered}
$$

whose discriminant factors as $47^{2} I(j, \mathcal{B})^{2} I\left(j, \mathcal{B}^{2}\right)^{2}$ with

$$
I(j, \mathcal{B})=5^{15} 11^{2} 13^{5} 19^{4} 23^{2} 29 \cdot 31 \cdot 41, \quad I\left(j, \mathcal{B}^{2}\right)=5^{15} 11^{7} 13^{5} 19 \cdot 23 \cdot 29 \cdot 31 \cdot 41 \cdot 43,
$$

where $\mathcal{B}$ is ideal class of the prime ideal $\mathfrak{p}_{2}=\left(2, \frac{1+\sqrt{-47}}{2}\right)$, and similarly the discrimi-

\begin{tabular}{|c|c|c|c|c|c|c|c|c|}
\hline$g$ & $f$ & $f^{2}$ & $f^{3}$ & $f^{4}$ & $f^{6}$ & $f^{8}$ & $f^{12}$ & $f^{24}$ \\
\hline$I(a$ & 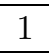 & 1 & 11 & 19 & $5 \cdot 11$ & $5^{2} \cdot 19$ & $5^{3} 11 \cdot 19$ & $5^{5} 11 \cdot 13^{2} 19 \cdot 23$ \\
\hline$(g, \mathcal{L})$ & 1 & 5 & 1 & 5 & 5 & $5 \cdot 31$ & $5^{3} 11$ & $5^{5} 11 \cdot 23 \cdot 31$ \\
\hline
\end{tabular}
nant of the polynomial $G\left(f^{r}, X\right)$ for $g=f^{r}$ with $r \mid 24$ factors as $47^{2} I(g, \mathcal{B})^{2} I\left(g, \mathcal{B}^{2}\right)^{2}$ with $I(g, \mathcal{A})$ given by:

To explain these numbers in terms of the formula explained in this section, we must look at each decomposition of $p$ in the form (16) with $\ell<p$ a prime quadratic nonresidue of $p$. There are $(p-1) / 2=23$ decompositions of $p$ as $B_{1}+B_{2}$ with $B_{1}, B_{2}>0,\left(B_{1} / p\right)=-1,\left(B_{2} / p\right)=+1$, and for each such decomposition a unique prime quadratic nonresidue $\ell$ of $p$ dividing $B_{1}$ to an odd power (since the smallest product of three prime nonresidues of $p$ is $715>p$ ) and various representations of $B_{1} / \ell$ and $B_{2}$ as norms of ideals of $K=\mathbb{Q}(\sqrt{-p})$. Rather than making a table of all cases, we give all the details for the prime $\ell=11$; the other primes $\ell$ work similarly and yield the numbers given in the above table.

The 25 decompositions of $p$ in the form (16) with $\ell=1$ all have $k=1$ and are given by

$$
\begin{array}{lll}
\mathfrak{b}_{1}=(1), & \mathfrak{b}_{2} \in\left\{\mathfrak{p}_{2}^{2} \mathfrak{p}_{3}^{2}, 2 \mathfrak{p}_{3}^{2}, \overline{\mathfrak{p}}_{2}^{2} \mathfrak{p}_{3}^{2}, 3 \mathfrak{p}_{2}^{2},(6), 3 \overline{\mathfrak{p}}_{2}^{2}, \mathfrak{p}_{2}^{2} \overline{\mathfrak{p}}_{3}^{2}, 2 \overline{\mathfrak{p}}_{3}^{2}, \overline{\mathfrak{p}}_{2}^{2} \overline{\mathfrak{p}}_{3}^{2}\right\} ; \\
\mathfrak{b}_{1} \in\left\{\mathfrak{p}_{2}, \overline{\mathfrak{p}}_{2}\right\}, & \mathfrak{b}_{2}=(5) ; \\
\mathfrak{b}_{1} \in\left\{\mathfrak{p}_{3}, \overline{\mathfrak{p}}_{3}\right\}, & \mathfrak{b}_{2} \in\left\{\mathfrak{p}_{2} \mathfrak{p}_{7}, \overline{\mathfrak{p}}_{2} \mathfrak{p}_{7}, \mathfrak{p}_{2} \overline{\mathfrak{p}}_{7}, \overline{\mathfrak{p}}_{2} \overline{\mathfrak{p}}_{7}\right\} ; \\
\mathfrak{b}_{1} \in\left\{\mathfrak{p}_{2}^{2},(2), \overline{\mathfrak{p}}_{2}^{2}\right\}, & \mathfrak{b}_{2} \in\left\{\mathfrak{p}_{3}, \overline{\mathfrak{p}}_{3}\right\},
\end{array}
$$

where $\mathfrak{p}_{3}$ and $\mathfrak{p}_{7}$ are prime ideals of norm 3 and 7 , respectively, and $\overline{\mathfrak{p}}_{p}(p=2,3,7)$ denote the conjugates of $\mathfrak{p}_{p}$. We can choose the ideals $\mathfrak{p}_{3}$ and $\mathfrak{p}_{7}$ to belong to the ideal classes $\mathcal{B}^{2}$ and $\mathcal{B}$, respectively, since $K$ contains integers of norm 12 and 14 . Then we find that of the 25 cases listed there are 7 for which $\mathfrak{b}_{2}$ is principal, 2 for which $\mathfrak{b}_{2}$ belongs to the class $\mathcal{B}$ (and of course equally many with $\mathfrak{b}_{2} \in \mathcal{B}^{4}$ ), and 7 with $\mathfrak{b}_{2} \in \mathcal{B}^{2}$ (or $\mathcal{B}^{3}$ ). This explains the powers $11^{2}$ and $11^{7}$ in the numbers $I(j, \mathcal{B})$ and $I\left(j, \mathcal{B}^{2}\right)$ given above.

Finally, of the 25 decompositions (16), there are only 5 with $\mathfrak{b}_{1} \mathfrak{b}_{2}$ divisible by 2 , and of these there are one each with $\mathfrak{b}_{2}$ belonging to the ideal classes $\mathcal{B}$ or $\mathcal{B}^{2}$. This explains why the exponent of 11 in $I\left(f^{24}, \mathcal{B}\right)$ and $I\left(f^{24}, \mathcal{B}^{2}\right)$ is 1 . For the decomposition having $\mathfrak{b}_{2} \in \mathcal{B}$, namely $\mathfrak{b}_{1}=(1), \mathfrak{b}_{2}=2 \overline{\mathfrak{p}}_{3}^{2}$, the ideal $\mathfrak{a}=\mathfrak{b}_{1} \mathfrak{b}_{2} / 2$ is primitive of norm 9, so the number occurring on the left-hand side of (13) is 104, which is divisible by $24 / r$ if and only if $r$ is a multiple of 3 , explaining why the factor $11^{1}$ occurs in $I\left(f^{r}, \mathcal{B}\right)$ in these cases. Similarly, for the decomposition for 
which $\mathfrak{b}_{2} \in \mathcal{B}^{2}$, namely $\mathfrak{b}_{1}=(2), \mathfrak{b}_{2}=\mathfrak{p}_{3}$, we have that $\mathfrak{a}$ is primitive of norm 3 , so the left-hand side of (13) is 38 , which is divisible by $24 / r$ only for $r=12$ and 24 .

\section{WEBER POLYNOMIALS FOR OTHER DISCRIMINANTS}

In theory one could repeat the analysis of the previous sections for discriminants congruent to $5(\bmod 8), 0(\bmod 4)$, or $0(\bmod 3)$, obtaining (conjectural) formulas for the conjugation behavior of the singular Weber moduli and for the norms of their differences. The results in general would be less sharp than in the case of discriminants satisfying (1) since, as mentioned in the introduction, one cannot usually get class invariants by going all the way from $j$ to $\mathfrak{f}$ but has to take a modular function on some intermediate group. We will restrict ourselves to giving some partial discussion of the two cases

A. $d \equiv 5(\bmod 8)($ so that 2 is inert) but still $\not \equiv 0(\bmod 3)$;

B. $d \equiv 1(\bmod 8)$ as before but now $3 \mid d$.

In both cases we will end up in small extension fields of the Hilbert class field $H=\mathbb{Q}(\sqrt{d})\left(j_{0}\right)$ and its real subfield $H^{+}=\mathbb{Q}\left(j_{0}\right), j_{0}=j\left(\frac{1+\sqrt{d}}{2}\right)$.

A. If $d \equiv 5(\bmod 8)$, then the correct class invariant is $\mathfrak{f}(\sqrt{d})$ rather than $\sqrt{2} / \mathfrak{f}(\sqrt{d})$ as before. It generates the same number field as $j_{0}=j(\sqrt{d})$ (this was conjectured by Weber [11, §127] and proved by Schertz [9]), but this field no longer coincides with $H^{+}$since now $h(4 d)$ equals $3 h(d)$ rather than $h(d)$ as in the case $d \equiv 1(\bmod 8)$. Instead, $\mathfrak{f}(\sqrt{d})$ satisfies a cubic equation of the form

$$
X^{3}-2 \lambda X^{2}+2 \mu X-2=0
$$

with $\lambda$ and $\mu$ integers in $H^{+}$. (In particular, $\mathfrak{f}(\sqrt{d})^{3} / 2$ is a unit.) The other two roots of this equation are $\sqrt{2} / \mathfrak{f}\left(\frac{ \pm b+\sqrt{d}}{2}\right)$ where $b \equiv 0(\bmod 3)$ and $\frac{b^{2}-d}{4} \equiv 1(\bmod 16)$, so the numbers $\lambda$ and $\mu$ can be obtained easily, using an analogue of the proposition in $\S 1$ to find the numerical values of their conjugates. These two numbers have relatively small height and generate $H^{+}$, so we again get reasonable equations for generating the class field. Some examples are given in Table 5. In all cases either $\theta=\lambda$ or $\theta=\mu$ (or $\theta=0$ if $h=1$ ) generates $H^{+}$, but the cases $d=-83$ and $d=-427$ show that neither $\lambda$ nor $\mu$ alone always works. We do not know whether there is a universal combination of $\lambda$ and $\mu$ which always generates the class field.

Note that, since the Galois group of $H / \mathbb{Q}$ is solvable (dihedral), we could have given a closed formula for the generator $\theta$ in the last column instead of its minimal polynomial over $\mathbb{Q}$. For instance, the $\theta$ for $d=-155$ has the minimal polynomial $X^{2}-\frac{1+\sqrt{5}}{2} X+\frac{1-\sqrt{5}}{2}$ over $\mathbb{Q}(\sqrt{5})$ (the real subfield of the genus field of $\mathbb{Q}(\sqrt{d})$ ) and is given explicitly by

$$
\theta=\frac{1+\sqrt{5}}{4}+\sqrt{\frac{-1+5 \sqrt{5}}{8}},
$$

while for $d=-107$ we have

$$
\theta=\frac{\sqrt[3]{6 \sqrt{3}+\sqrt{107}}+\sqrt[3]{6 \sqrt{3}-\sqrt{107}}}{\sqrt{3}}
$$

B. We now consider the case when $d \equiv 1(\bmod 8)$ but $3 \mid d$. Then the class number $h$ of $\mathbb{Q}(\sqrt{d})$ is divisible by 2 , the Hilbert class field $H$ contains the biquadratic field $\mathbb{Q}(\sqrt{d}, \sqrt{-3})$, and its subfield $H^{+}$has degree $h / 2$ over the real quadratic field $\mathbb{Q}(\sqrt{-d / 3})$. Let $\varepsilon$ be the fundamental unit of this latter field. The number $\alpha=$ 
TABLE 5. Class fields for discriminants $d \equiv 5(\bmod 8)$

\begin{tabular}{ccccc}
$|d|$ & $h(d)$ & $\lambda$ & $\mu$ & minimal polynomial of $\theta \in H^{+}$ \\
\hline 11 & 1 & 1 & 1 & $X$ \\
19 & 1 & 0 & -1 & $X$ \\
35 & 2 & $\theta$ & $\theta$ & $X$ \\
43 & 1 & 1 & 0 & $X^{2}-X-1$ \\
59 & 3 & $\theta$ & $\theta^{2}-\theta$ & $X$ \\
67 & 1 & 1 & -1 & $X^{3}-X^{2}-3 X+4$ \\
83 & 3 & 1 & $\theta$ & $X^{2}+X-3$ \\
91 & 2 & 1 & $\theta$ & $X^{3}-X-4$ \\
107 & 3 & $\theta$ & $\left(\theta^{2}-\theta-2\right) / 2$ & $X^{2}-3 X+1$ \\
115 & 2 & $\theta$ & $\theta$ & $X^{3}-X^{2}-2 X-4$ \\
131 & 5 & $\theta$ & $\left(-\theta^{3}+\theta^{2}-\theta+2\right) / 2$ & $X^{5}-2 X^{4}+X^{3}-4$ \\
139 & 3 & $\theta$ & $\left(\theta^{2}-\theta-2\right) / 2$ & $X^{4}-X^{3}-3 X-1$ \\
155 & 4 & $\theta$ & $-1-\theta$ & $X$ \\
163 & 1 & 3 & 2 & $X^{2}-3 X-2$ \\
179 & 5 & $\theta$ & $\left(\theta^{2}-\theta-2\right) / 2$ & $X^{5}-5 X^{3}-16 X^{2}-16 X-8$ \\
187 & 2 & $\theta$ & $\theta$ & $X^{3}-X-1$ \\
211 & 3 & $\theta$ & $-\theta$ & $X^{2}+5 X+3$ \\
235 & 2 & $-\theta+3$ & $\theta$ & $X^{2}-7 X-3$ \\
403 & 2 & $-2 \theta-2$ & $\theta$ &
\end{tabular}

$\sqrt{2} / \mathfrak{f}(\sqrt{d})$ has degree $3 h$ rather than $h$ over $\mathbb{Q}$ and only its cube lies in $H^{+}$. However, in all the examples we looked at, it was the case that (for an appropriate choice of $\varepsilon)$

$$
\alpha^{3 h} / \varepsilon=\text { cube (of a unit) in } H^{+} .
$$

Suppose that this is true. Then we have the following two possibilities:

i) If the class number $h$ is $\equiv \pm 1(\bmod 3)$, then $(17$ ? $)$ implies that $\alpha / \varepsilon^{ \pm 1 / 3}$ belongs to $H^{+}$, so again we get relatively small generators of the class field. As an example, take $d=-159$, with $h=10$. Then the minimal polynomial of $\alpha^{3}$ over $\mathbb{Q}$ is

$$
X^{10}-X^{9}+X^{8}+7 X^{7}+63 X^{6}+121 X^{5}+219 X^{4}+196 X^{3}+146 X^{2}+47 X-1,
$$

with discriminant $3^{12} 11^{2} 17^{4} 53^{5} 59^{2} 79^{2}$, while that of $\alpha / \varepsilon^{1 / 3}$, where $\varepsilon=(7+\sqrt{53}) / 2$, is

$$
X^{10}-3 X^{9}+7 X^{8}-2 X^{7}+15 X^{6}+18 X^{5}-37 X^{4}-60 X^{3}-27 X^{2}-2 X+1
$$

with discriminant $3^{8} 53^{5} 79^{2} 223^{2}$, which is somewhat better. However, the discriminant of $H^{+}$is $3^{4} 53^{5}$ and this field contains an element with the yet simpler minimal polynomial

$$
X^{10}-X^{9}+2 X^{8}+7 X^{7}+X^{6}-15 X^{5}-5 X^{4}+8 X^{3}+5 X^{2}-5 X+1
$$

of discriminant $3^{8} 19^{2} 53^{5}$, so the polynomials obtained from $\alpha^{3}$ and $\alpha / \varepsilon^{1 / 3}$ are - as is to be expected - not as good as the ones obtained directly from $\alpha$ in the case $3 \nmid d$.

ii) On the other hand, if $h$ is divisible by 3 , then (17?) no longer lets us obtain an equation of degree $h$ for $\alpha$ by dividing by $\varepsilon^{ \pm 1 / 3}$. Instead, it says that $H^{+}$contains the sextic field $\mathbb{Q}\left(\varepsilon^{1 / 3}\right)$. Then $\alpha^{3}$, which has degree $h$ over $\mathbb{Q}$, can also be given by 
an equation of degree $h / 2$ over $\mathbb{Q}(\varepsilon)$ and by an equation of degree $h / 6$ over $\mathbb{Q}\left(\varepsilon^{1 / 3}\right)$. For instance, the generator $x=(\sqrt{2} / f(\sqrt{d}))^{3}$ of $H^{+}$for $d=-87$ (class number 6 ) satisfies the equations

$$
\begin{aligned}
& x^{6}+x^{5}+4 x^{4}-4 x^{3}+11 x^{2}+13 x-1=0, \\
& x^{3}-\left(2+\varepsilon^{-1}\right) x^{2}+\left(3-\varepsilon^{-1}\right) x-\varepsilon^{-1}=0, \\
& 3 x+\left(2 \varepsilon^{5 / 3}+\varepsilon^{4 / 3}+\varepsilon-10 \varepsilon^{2 / 3}-7 \varepsilon^{1 / 3}-3\right)=0
\end{aligned}
$$

where $\varepsilon=(5+\sqrt{29}) / 2$. Similarly, the generator $x$ for $d=-231(h=12)$ satisfies

$$
3 x^{2}-\left(6 \eta^{5}-\eta^{4}-53 \eta^{2}+9 \eta-2\right) x+\left(2 \eta^{5}-2 \eta^{4}+\eta^{3}-19 \eta^{2}+17 \eta-2\right)=0
$$

where $\eta=\varepsilon^{1 / 3}, \varepsilon=(9+\sqrt{77}) / 2$.

\section{ACKNOWLEDGEMENTS}

The first author was partially supported by a Natural Sciences and Engineering Research Council of Canada grant, as well as Senior Research Fellowships at Newnham College and the Department of Pure Mathematics and Mathematical Statistics, University of Cambridge, and by the Newton Institute. The paper was completed at the Max-Planck-Institut für Mathematik, Bonn.

\section{REFERENCES}

1. Atkin, O. and Morain, F., Elliptic curves and primality proving, Math. Comp. 61 (1993), 29-68. MR 93m:11136

2. Berwick, W.E.H., Modular invariants expressible in terms of quadratic and cubic irrationalities, Proc. London Math. Soc. 28 (1928), 53-69.

3. Birch, B., Weber's class invariants, Mathematika 16 (1969), 283-294. MR 41:6816

4. Cohn, H., Introduction to the Construction of Class Fields, Cambridge studies in advanced mathematics 6, Cambridge University Press, 1985. MR 87i:11165

5. Cox, D., Primes of the form $x^{2}+n y^{2}$, John Wiley \& Sons, 1989. MR 90m:11016

6. Deuring, M., Teilbarkeitseigenschaften der singulären Moduln der elliptischen Funktionen und die Diskriminante der Klassengleichung, Comm. Math. Helvetici 19 (1946), 74-82. MR 8:318d

7. Gross, B. and Zagier, D., On singular moduli, J. Reine Angew. Math. 355 (1985), 191-220. MR 86j: 11041

8. Kaltofen, E., and Yui, N., Explicit construction of the Hilbert class fields of imaginary quadratic fields by integer lattice reduction, Number Theory, New York Seminars 1989-90, Springer-Verlag, 1991, pp. 149-202. MR 92j:11133

9. Schertz, R., Die singulären Werte der Weberschen Funktionen $\mathfrak{f}, \mathfrak{f}_{1}, \mathfrak{f}_{2}, \gamma_{2}, \gamma_{3}$, J. Reine Angew. Math. 286/287 (1976), 46-74. MR 54:10205

10. Watson, G.N., Singular moduli (3), Proc. London Math. Soc. 40 (1936), 83-142.

11. Weber, H., Lehrbuch der Algebra, Bd. III, Braunschweig, 1908.

12. Hajir, F. and Rodriguez Villegas, F., Explicit elliptic units I, preprint, 1995.

Department of Mathematics, Queen's University, Kingston, Ontario, Canada K7L $3 \mathrm{~N} 6$

E-mail address: yui@ny.mast.queensu.ca

Max-Planck-Institut für Mathematik, Gottfried-Claren-Strasse 26, 53225 Bonn, Germany

E-mail address: zagier@mpim-bonn.mpg.de 